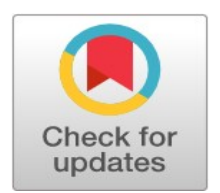

\title{
Automated age estimation based on geometric mean projection transform using Orthopantomographs
}

\author{
Seyed Mostafa Mousavi Kahaki ${ }^{1 *}$, Waidah Ismail ${ }^{2}$, Md Jan Nordin ${ }^{3}$, Nazatul S. Ahmad ${ }^{4}$, \\ Marina Ahmad 5
}

${ }^{1,3}$ Center for Artificial Intelligence Technology, National University of Malaysia, Bangi, Malaysia

${ }^{2,5}$ Faculty of Science and Technology, Universiti Sains Islam Malaysia, Nilai, Malaysia

${ }^{4}$ Faculty of Dentistry, Universiti Sains Islam Malaysia, Kuala Lumpur, Malaysia

\author{
Index Terms \\ Orthopantomographs \\ Dental Image Analysis \\ Image Transform \\ Age Estimation \\ Received: 4 June 2016 \\ Accepted: 10 July 2016 \\ Published: 12 February 2017
}

\begin{abstract}
A new age assessment technique based on proposed geometric mean transform by using dental X-Ray Imagery analysis has been proposed in this study. Topology segmentation and content-based shape analysis by using image transforms have been proposed to extract the feature vectors of dental imagery. Then, the accumulated feature vectors have been classified to age classes by using Artificial Neural Networks (ANN). The proposed technique has been evaluated in the standard images sourced from faculty of dentistry, USIM, Malaysia. The evaluation results indicate the robustness of the technique to different types of dental images available in the dataset.
\end{abstract}

(C) 2017 The Author(s). Published by TAF Publishing.

\section{INTRODUCTION}

In forensic medicine, dental age plays an important role to assist in estimating the chronological age (CA) of a child. Chronological age is typically defined as an age calculated based on birth date. Estimation of child's age is crucial when the CA is missing or unrecorded. In [1] they reported only half of children in developing world register their birth. This gives us significant sign that age estimation is vital especially when involving forensic investigation and judicial proceeding. In addition, age estimation is also important for clinical dentistry to diagnose and plan for treatment as well as in other areas of dentistry [2] and [17]. Several biological ages have been developed: skeletal age, morphological age, secondary sex character age and dental age. These criteria can be applied separately or together to assess the degree of physiological maturity of growing child [3]. Dental age based on tooth development stages was first introduced by [3] and is considered the most simple and reliable method as it has the highest value for both intra- and inter- observer agreement [4].

Demirjian and his colleagues introduce a system of age estimation based on tooth development stages. They classified teeth development in eight stages which are marked from A-H and 0 stage for no appearance. Their classification also separates between boys and girls. Each stage is given a rating and then these ratings are summed-up to a final score called maturity score. They provide a table which is used to translate the maturity score into age. Automated system is very crucial in aiding the experts in identifying CA of a patient based on dental features. With automated system, it may reduce time and error compared to naked eyes' observation. The source of teeth data comes from X-ray dental film. The X-ray is a cheap and very useful tool for dentist diagnosis. It has a low level of radiation, is comfortable and very quick for the patient to have taken. However, X-ray images suffer degradation from low resolu-

\footnotetext{
* Corresponding author: Seyed Mostafa Mousavi Kahaki

†Email: kahaki@ukm.edu.my
} 
tion which contributes to the existence of noise in the images. One of the essential steps to process dental X-ray images is to distinguish between Region of Interest (ROI) and the background. This task can be carried out through image segmentation. Therefore, image segmentation of X-ray images faced big challenges due to the poor quality of X-ray images.

The main task in image segmentation is to divide an image into its constitute regions or objects. Segmentation is recognition and labeling of distinct tooth in the X-ray image. Object obtained from the image characterizes ROI containing significant information considered for later process. ROI can be defining as an amount of image that emphasis on single object. In most of the segmentation algorithms, the segmentation is done either by extracting region-based features that can identify different objects and regions, or by applying a model and trying to adjust its parameters to fit the processed objects or regions.

Basically, segmentation of dental X-ray images may be classified into three basic techniques; pixel-based segmentation, region-based segmentation and boundarybased segmentation which are introduced by [5]. While some other literature gives further category of segmentation generalizing on X-ray image segmentation such as wavelet-based pattern recognition-based, deformable models, wavelets-based methods, and atlas-based techniques given by [6].

The simplest method and typically used in image segmentation is by using thresholding method and a popular threshold method was invented by [7]. Pixels in grayscale image are separated into two classes; either the pixels are below the threshold value or above threshold value. This technique can be extended to multiple threshold values to suit more types of images such as multi-color images. Otsu technique is utilized to eliminate background from a picture. However, selecting a threshold value is challenging and it will determine whether an image is over segment or otherwise.

\section{RELATED LITERATURE}

In dental X-ray image segmentation, [5] use the level set method for doing boundary-based segmentation. This method detects edges and angles and other image characteristics on surface which is covered by a curve. However, in their research [8], they found that selecting a proper function that characterizes the curve is a big issue and still many researches have to be done to answer the question. Regionbased method is also a method for segmentation of image of dental X-ray. This method grouped pixels based on similarities of some criteria chosen. The pre-defined criteria may come from color, gray-level, texture, etc. A number of seed points are utilized in the division process to ensure the spatial constraints. It seems difficult to regulate the important coefficient for grouping the pixels. Furthermore, it better functionality is dependent upon prefix function of curves are provided [9]. Even other image processing techniques like thresholding, boundary-based and also regionbased techniques also face difficulty while determination of parameters for teeth sample but they provide reasonable accuracy [10].

In [11], a new watershed algorithm, which may be used in segmentation of proposed dental X-ray images. Formal watershed algorithm's over-segmentation issue is considered by the researchers in order to resolve. Top-hatbottom-hat transformation has been used to intensify the contrast of front, background and eliminate noises. After that erosion process has been applied to wane the level of adhesion between teeth. By using Hole-filling also can help to eliminate some unnecessary split-line which can cause the phenomenon of wrong segmentation. I order to get the results of segmentation watershed algorithm has been used.

[12] Proposed a new cooperative scheme that applies semi-supervised fuzzy clustering algorithms to dental $\mathrm{X}$-ray image. The Otsu technique is utilized to erase background area from the images of dental X-ray. Then, FCM has been considered eliminate range of dental structure from the obtained results of last procedure. Finally, eSFCM is used as robust technique in order to confirm the findings of last step. The authors remark that their framework does not use any dental features in their algorithm of clustering.

The authors remark that their framework does not use any dental features in their algorithm of clustering. Using the reconstructed images, the tooth and Pulp-Tooth Volume Ratios (PTVR) at four levels (crown area (L1); coronal one third (L2); midroot (L3), and apical one third of the root (L4)) were measured [13].

\section{METHOD}

Based on the shortcomings of the available methods, a new method to segment and classify the tooth images based on the 3rd molar in order to achieve age estimation is proposed in this section. The stages of the proposed age estimation method based on 3rd molar in Orthopantomographs are shown in Figure 1. 


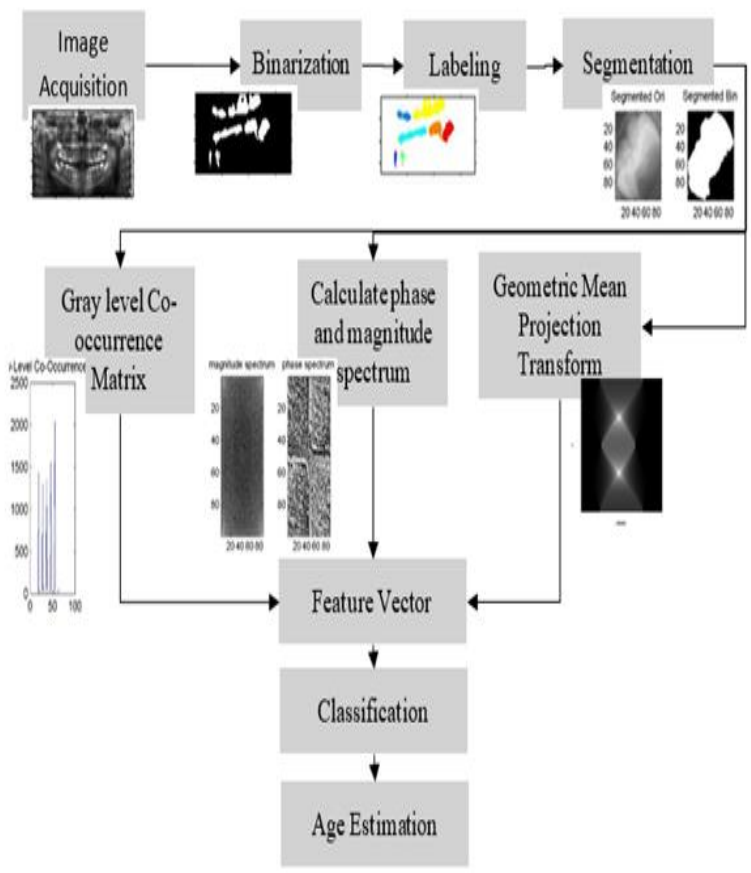

Fig. 1. Proposed age estimation method based on 3rd molar

In the first step, orthopantomography radiology images are captured and converted to binary images. In order to segment the image to extract the region of interest, the binary image labeled using image labeling technique and the 3rd polar is extracted as the ROI to extract the features. The feature vector is calculated by using cooccurrence metric, phase and magnitude spectrum and the proposed geometric mean projection transform. This new image transform form on geometric mean properties of integral values in horizontal as well as vertical image commands.

The new transform is based on geometric mean projection, which - unlike other image transforms, such as Radon transform - does not consider all signals in an image with the same signal acquisition rate. Instead, it is general and thus applicable to all capturing signal functions to achieve sufficient invariant features. The Geometric Mean Projection Transform (GMPT) which is based on Mean Projection Transform (MPT) [14] guarantees that the detector only extracts the highly informative information from the shape to achieve an invariant feature vector for an accurate classification process. The GMPT is an image transforms that containing integrals of geometric mean over straight lines in a digital image. Considering as the function of the image signal in, then GMPT is a function transform of , where the geometric mean of the horizontal and vertical integral is calculated by using Equation (1).

$$
G M P T=\prod_{i=1}^{n}\left(\int_{S x} F(x, y)|d x|, \int_{S y} F(x, y)|d y|\right)
$$

To parametrize of any signal with respect to the arclength and the Euclidean distance from the origin to , GMPT can be written as:

$(x(t), y(t))=\prod[(t \sin (\alpha)+\operatorname{scos}(\alpha),(-t \cos (\alpha)+\sin (\alpha))]$

Where $\alpha$ is the angle of the vector, and $(\alpha, s)$ represents transform bounds on $R^{2}$ for entire signals, and GMPT may be characterized in the said coordinates as per to Equation (3):

$G M P T=\prod_{i=1}^{n}\left(\int_{-\infty}^{+\infty} F(x(t), y(t))|d x|, \int_{-\infty}^{+\infty} F(x(t), y(t))|d y|,\right)$

(3)

Which can also be written as:

$G M P T=\prod_{i=1}^{n}\left(\int_{-\infty}^{+\infty} F(t \sin (\alpha)+\operatorname{scos}(\alpha)) \mid d x\right), \int_{-\infty}^{+\infty}(-t \cos (\alpha)$

$+\sin (\alpha))|d y|)(4)$

The GMPT calculates the geometric mean of the integrals of an input image function in vertical and horizontal directions to calculate the feature vector matrix of each image. The result of feature vector calculated by using GMPT transform is presented in Figure 2. 


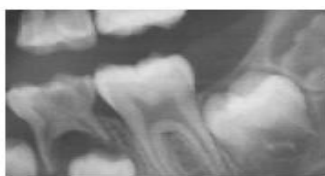

(a)

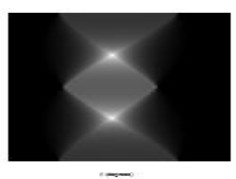

(b)
Fig. 2 . (a) Dental X-Ray segmented image, (b) GMPT transform result

\section{RESULTS AND DISCUSSION}

In order to evaluate the result, a new dataset called Malaysian Children Dental Development (MCDD) [15] and [16] dataset is developed as a part of this research. MCDD contains X-Ray images and the image information of 456 patients collected from Malaysian children aged 1 to 17 years old from the collection available at the faculty of the dentistry research at Universiti Sains Islam Malaysia. The information related to age and sex distribution of the collected data is shown in Table 1. The information related to age and sex distribution of the collected data is shown in Table 1.

TABLE 1

DATA POPULATION INFORMATION

\begin{tabular}{lll}
\hline \hline Age & & 3rd molar \\
\hline & Male & Female \\
\hline $1-5$ & 22 & 22 \\
$6-10$ & 107 & 150 \\
$11-15$ & 67 & 66 \\
$>16$ & 11 & 11 \\
Total & 207 & 149 \\
\hline \hline
\end{tabular}
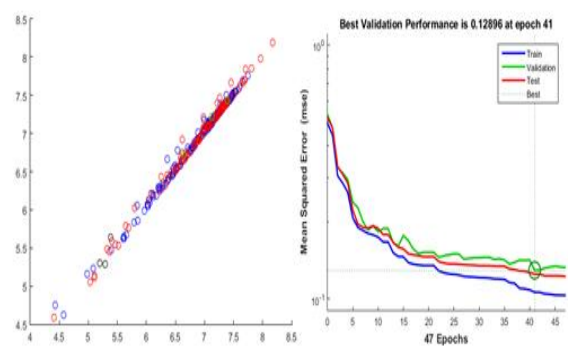

Fig. 3. Classification result. a) features' estimation, b) validation performance

3rd molars were selected for classification after the segmentation stage in this study as they are more reli- able in this study population case. Simpler stages for segmentation and classification in collected data make us to come to the conclusion that the $3 \mathrm{rd}$ molar has a great level of possibility as a forensic sample data for estimating the age. Figure 3 depicted the classification result and performance graph using ANN algorithm.

Table 2 presents the performance result of the classification by using ANN for different classes. The result is also separated based on the male and female population and the average. The result of classification by using the proposed method indicates an acceptable result.

TABLE 2

PERFORMANCE RESULT IN DIFFERENT AGE CLASSES

\begin{tabular}{lll}
\hline \hline Class (teeth stage) & & Performance \\
\hline & Male & Female \\
\hline PTVR1 & $74 \%$ & $76 \%$ \\
PTVR2 & $78 \%$ & $79 \%$ \\
PTVR3 & $84 \%$ & $83 \%$ \\
PTVR4 & $83 \%$ & $81.5 \%$ \\
Mean & $79.75 \%$ & $79.87 \%$ \\
Average & & $79.81 \%$ \\
\hline \hline
\end{tabular}

\section{CONCLUSION}

Combination of the co-occurrence metric, phase and magnitude spectrum and the proposed geometric mean projection transform to extract and classify the 3rd molar teeth in orthopantomography radiology images indicates an acceptable classification performance for age estimation. The result of classification for different root stages demonstrates higher rate for final stages of the teeth. The average result indicates that the proposed method is a reliable method for age estimation in the Malaysian children population for pulp-tooth volume measurements and age estimation.

\section{ACKNOLEDGEMENT}

The authors would like to thank the Ministry of Education for the research sponsorship funds under grant no. USIM/FRGS/FST/32/51513 and DIP-2016-018. We also

appreciate Center for Artificial Intelligence Technology (CAIT) at National University of Malaysia and Faculty of Dentistry, Universiti Sains Islam Malaysia for the X-ray images and the laboratory facilities. 


\section{REFERENCES}

[1] S. Terry and B. Laura, "Age assessment practices: A literature review \& annotated bibliography," United Nations Children's Fund, New York, NY, 2011.

[2] M. Maber, H. M. Liversidge and M. P. Hector, "Accuracy of age estimation of radiographic methods using developing teeth," Forensic Science International, vol. 159, S68-S73, 2006. D0I: 10.1016/j.forsciint.2006.02.019

[3] A. Demirjian, H. Goldstein and J. M. Tanner, "A new system of dental age assessment," Human Biology, vol. 45, no. 2, pp. 211-227, 1973.

[4] A. Olze, W. Reisinger, G. Geserick and A. Schmeling, "Age estimation of unaccompanied minors: Part II. Dental aspects," Forensic Science International, vol. 159, pp. S65-S67, 2006. DOI: 10.1016/j.forsciint.2006.02.018

[5] R. E. Rad, M. S. Mohd Rahim, A. Rehman, A. Altameem and T. Saba, "Evaluation of current dental radiographs segmentation approaches in computer-aided applications," IETE Technical Review, vol. 30, no. 3, pp. 210-222, 2013. DOI: $10.4103 / 0256-4602.113498$

[6] C. Stolojescu-CriŞan and C. Holban, "A comparison of X-Ray image segmentation techniques," Advances in Electrical and Computer Engineering Engineering, vol. 13, no. 3, pp. 85-92, 2013. DOI: 10.4316/AECE.2013.03014

[7] N. Otsu, "A threshold selection method from gray-level histograms," Automatica, vol. 11, no. 285-296, pp. 23-27, 1975.

[8] R. E. Rad, A. E., M. S. M. Rahim and A. Norouzi, "Level set and morphological operation techniques in application of dental image segmentation," World Academy of Science, Engineering and Technology, International Journal of Medical, Health, Biomedical, Bioengineering and Pharmaceutical Engineering, vol. 8, no. 4, pp. 182-185, 2014.

[9] E. H. Said, D. E. M. Nassar, G. Fahmy and H. H. Ammar, "Teeth segmentation in digitized dental X-ray films using mathematical morphology," IEEE Transactions on Information Forensics and Security, vol. 1, no. 2, pp. 178-189, 2006. DOI: 10.1109 /TIFS.2006.873606

[10] G. E. Sujji, Y. V. S. Lakshmi and G. W. Jiji, "MRI brain image segmentation based on thresholding," International Journal of Advanced Computer Research, vol. 3, no. 1, pp. 97-101, 2013.

[11] H. Li, G. Sun, H. Sun and W. Liu, "Watershed algorithm based on morphology for dental X-ray images segmentation," Paper presented at the IEEE 11th International Conference on Signal Processing (ICSP), Beijing, China, 2012.

DOI: $10.1109 /$ icosp.2012.6491720

[12] T. M Tuan, "A cooperative semi-supervised fuzzy clustering framework for dental X-ray image segmentation," Expert Systems with Applications, vol. 46, pp. 380-393, 2016. DOI: 10.1016/j.eswa.2015.11.001

[13] H. Aboshi, T. Takahashi and T. Komuro, "Age estimation using microfocus X-ray computed tomography of lower premolars," Forensic Science International, vol. 200, no. 1, pp. 35-40, 2010. DOI: 10.1016/j.forsciint.2010.03.024

[14] S. M. M. Kahaki, M. J. Nordin and A. H. Ashtari, "Contour-based corner detection and classification by using mean projection transform," Sensors, vol. 14, no. 3, pp. 4126-4143, 2014. DOI: 10.3390/s140304126

[15] S. M. M. Kahaki, M. J. Nordin and A. H. Ashtari, "Contour-based corner detection and classification by using mean projection transform," Sensors, vol. 14, no. 3, pp. 4126-4143, 2014. DOI: 10.3390/s140304126

[16] S. M. M. Kahaki, W. Ismail, M. J.Nordin and N. S. Ahmand, "Malaysian Children Dental Development (MCDD)," [Online]. Available: research.ftsm.ukm.my/kahaki/MCDD.zip

[17] T. Dokhan, N. Shebani and A. Zaet, "Relationship of bolton's ratios and tooth-size discrepancy," Journal of Advances in Health and Medical Sciences, vol. 2, no. 1, pp. 34-41, 2016. DOI: 10.20474/jahms2.1.5

— This article does not have any appendix. - 